\title{
Stromal cell-derived factor-1 promotes survival of pancreatic beta cells by the stabilisation of beta-catenin and activation of transcription factor 7-like 2 (TCF7L2)
}

\author{
Z. Liu • J. F. Habener
}

Received: 18 December 2008 / Accepted: 3 April 2009 /Published online: 26 May 2009

(C) Springer-Verlag 2009

\begin{abstract}
Aims/hypothesis Stromal cell-derived factor-1 (SDF-1) is a chemokine produced in stromal tissues in multiple organs. Earlier we reported on levels of SDF-1 and SDF-1 receptor (CXCR4) in the insulin-producing beta cells of the mouse pancreas and determined that the SDF-1/CXCR4 axis is important for beta cell survival through activation of the prosurvival kinase, protein kinase B (AKT). Since AKT is known to modulate the wingless-type MMTV integration site family (WNT) signalling cascade, we examined the effects of SDF-1/CXCR4 on WNT signalling in beta cells and whether this signalling is important for cell survival. Methods Activation of downstream WNT signalling (beta-catenin and transcription factor 7-like 2, [TCF7L2]) in response to SDF-1 was examined in the islets of WNT signalling reporter (Tcf-optimal promoter betagalactosidase) mice and in INS-1 and MIN6 beta cells. Cytoprotection of beta cells by SDF-1 in response to the induction of apoptosis was assessed by caspase 3 and TUNEL assays.

Results SDF-1 induced WNT signalling in beta cells of isolated islets and in INS-1 cells via CXCR4-mediated activation of Galphai/o-coupled signalling and the phosphatidylinositol 3-kinase/AKT signalling cascade resulting in the inhibition of glycogen synthase kinase 3-beta. The
\end{abstract}

Electronic supplementary material The online version of this article (doi:10.1007/s00125-009-1384-x) contains supplementary material, which is available to authorised users.

Z. Liu · J. F. Habener $(\bowtie)$

Thier 306, Laboratory of Molecular Endocrinology,

Massachusetts General Hospital and Harvard Medical School,

55 Fruit Street,

Boston, MA 02114, USA

e-mail: jhabener@partners.org key WNT signalling regulators, beta-catenin and AKT, were activated by SDF-1 at the transcriptional and posttranslational levels. Specific inhibition of beta-catenin in the WNT signalling cascade reversed the anti-apoptotic effects of SDF-1.

Conclusions/interpretation SDF-1 promotes pancreatic beta cell survival via activation of AKT and downstream WNT signalling via the stabilisation and activation of betacatenin/TCF7L2 transcriptional activators. These findings suggest a mechanism for SDF-1 based glucose-lowering therapies by enhancing beta cell mass through increasing cell survival.

Keywords Beta cells · Apoptosis · Diabetes · SDF-1 · WNT signalling
Abbreviations
AKT Protein kinase B
CXCR4 Stromal cell-derived factor-1 receptor
ERK Extracellular signal-regulated kinase
GLP-1 Glucagon-like peptide 1
GLP-1R GLP-1 receptor
GPCR G-protein-coupled receptor
GSK3 Glycogen synthase kinase 3
hIPC Human islet-derived precursor cell
PARP Poly ADP ribose polymerase
PI3K Phosphatidylinositol 3-kinase
PKA Protein kinase A
SDF-1 Stromal cell-derived factor-1
siRNA Small interfering RNA
TCF7L2 Transcription factor 7-like 2
TCF/LEF T-cell-specific transcription factor/lymphoid enhancer binding factor
TOPGAL Tcf-optimal promoter beta-galactosidase
WNT Wingless-type MMTV integration site family 


\section{Introduction}

Diabetes results from inability of the beta cells of the endocrine pancreas (islets of Langerhans) to produce insulin in amounts sufficient to maintain nutrient homeostasis. In both type 1 and type 2 diabetes beta cell mass is reduced and the remaining beta cells are stressed by the glucotoxic effects of prolonged, sustained hyperglycaemia [1]. Because a common feature of diabetes is a reduction in beta cell mass, an understanding of the factors and cellular mechanisms that control beta cell growth and survival may lead to new treatments for diabetes that enhance beta cell mass. We recently described the expression of the chemokine stromal cell-derived factor-1 Sdf1 and SDF-1 receptor (Cxcr4) on pancreatic beta cells [2]. The SDF-1/CXCR4 axis exerts cytoprotective effects on beta cells by activating the prosurvival kinase, protein kinase B (AKT), which in streptozotocin-induced diabetic mice results in attenuation of diabetes [2].

SDF-1 is a chemokine originally identified as a bone marrow stromal cell-secreted factor and now recognised to be produced in stromal tissues in multiple organs [3]. CXCR4 is highly specific for SDF-1. The most extensively studied function of the SDF-1/CXCR4 axis is that of chemoattraction involved in leucocyte trafficking and stem cell homing, in which local tissue gradients of SDF-1 attract circulating stem/progenitor cells [4]. The SDF-1/ CXCR4 axis is also involved in many aspects of development, cell survival and tissue repair and regeneration, as well as cancer $[5,6]$. The importance of SDF-1 in stem and progenitor cell recruitment has been established by observations that selective expression of SDF1 (also known as CXCL12) in injured tissues correlates with adult stem cell recruitment and tissue regeneration [7, 8]. Disruption of either $S d f 1$ or $C x c r 4$ genes in mice results in late embryonic lethality with multiple, generalised developmental defects in organogenesis $[9,10]$.

SDF-1/CXCR4 signalling in the pancreas remains relatively unexplored. WNT signalling in the pancreas has been reviewed recently [11]. A role for SDF-1 in the modulation of WNT signalling in the pancreas has not been reported to date. Sarvetnik and co-workers reported production of SDF-1 and CXCR4 in the fetal mouse pancreas and of CXCR4 in the proliferating duct epithelium of the regenerating pancreas in non-obese diabetic mice [12]. They also found that SDF-1 stimulates phosphorylation of AKT, ERK1/2 and sarcoma kinase (SRC), increases migration and decreases apoptosis of duct cells in vitro. Recent studies indicate that transgenic mice $\mathrm{p} \mathrm{SDF-1} \mathrm{in}$ their beta cells (RIP-SDF-1 mice) are protected from streptozotocin-induced diabetes by activation of the prosurvival protein kinase AKT and of resulting downstream prosurvival, anti-apoptotic signalling pathways [2]. Inter- estingly, the gene encoding SDF-1 is located near the type 1 diabetes susceptibility locus IDDM10, suggesting a contribution by SDF-1 to the induction of diabetes [13]. SDF-1 genetic polymorphisms are associated with the early onset of autoimmune diabetes [14]. Moreover, SDF-1/CXCR4 signalling is reported to confer anti-diabetogenic actions in the non-obese diabetic mouse model of autoimmune type 1 diabetes by the recruitment of Th2 cells [15].

The wingless-type MMTV integration site family (WNT) signalling cascade controls several cellular functions, including differentiation, proliferation and migration. The WNT proteins form a large family of cell-secreted factors that control diverse aspects of development [16-21]. WNT ligands exert their effect by binding to cell surface frizzled receptors and modulate the expression of various target genes through a series of intracellular processes ultimately leading to regulation of transcription [16-21]. The downstream WNT signalling pathway involves beta-catenin, which when stabilised translocates to the nucleus, where it associates with the T-cell-specific transcription factor/ lymphoid enhancer binding factor (TCF/LEF) family of transcription factors to regulate expression of canonical WNT target genes. In pancreatic beta cells, transcription factor 7-like 2 (TCF7L2) is a major form of transcription factor involved in the downstream WNT signalling that is responsible for activating growth-promoting genes in response to glucagon-like peptide-1 (GLP-1) agonists [22]. Notably, TCF7L2 has recently been found to be a major susceptibility factor for the development of type 2 diabetes manifested by diminished insulin production [23].

To date one single report implicates crosstalk of SDF-1 signalling with WNT signalling [24]. Stimulation of rat neural progenitor cells by SDF-1 results in the cytoplasmic accumulation of beta-catenin and concomitant nuclear translocation through a CXCR4 receptor-mediated ERK activation pathway. These findings prompted us to examine whether the SDF-1/CXCR4 axis regulates WNT signalling in pancreatic beta cells and whether WNT signalling is involved in the prosurvival actions of SDF-1. We examined SDF-1-activated WNT signalling in isolated islets and in INS-1 cells using a beta-catenin/TCF-activated reporter gene assay. We found that SDF-1 enhances WNT signalling through the Galphai/o-phosphatidylinositol 3-kinase (PI3K)-AKT axis, suppression of glycogen synthase kinase 3 (GSK3) $\beta$ and stabilisation of beta-catenin. We also provide evidence that active WNT signalling mediates prosurvival functions of SDF-1 on pancreatic beta cells.

\section{Methods}

Isolated mouse pancreatic islets Mouse islets were isolated [25] from the pancreases of Tcf-optimal promoter beta- 
galactosidase (TOPGAL) reporter mice transgenic for the LEF-LacZ WNT signalling reporter [26]. Freshly isolated islets were treated for $4 \mathrm{~h}$ with SDF-1 with and without the addition of the Galphai/o inhibitor pertussis toxin or the CXCR4 antagonist AMD3100. Beta-galactosidase activity was determined by incubation of the islets with X-gal for $6 \mathrm{~h}$. All mouse studies were approved by and in compliance with the MGH Institutional Animal Care Utilization Committee.

Polymerase chain reaction analyses of beta-galactosidase $m R N A$ levels in isolated mouse islets Islets from TOPGAL mice were removed after treatments with SDF-1, SDF-1 + AMD3100 and SDF-1+pertussis toxin. RNA was extracted and beta galactosidase mRNA levels were measured by PCR using a kit (SYBR Green QPCR; Stratagene, La Jolla, CA, USA) with the primers as described [22].

WNT signalling luciferase reporter assay (TOPflash) INS-1 cells were plated into 24-well dishes for $24 \mathrm{~h}$ before transfection with TOPflash (gift from R. Moon, Stanford University, CA, USA) or the mutant, control reporter FOPflash (gift from R. Moon, Stanford University, CA, USA) (1 $\mu \mathrm{g} /$ well) using LipofectAMINE2000 (Invitrogen, San Diego, CA, USA). Various concentrations of SDF-1 or AMD3100 were then added to the culture medium at $24 \mathrm{~h}$ following transfection for the indicated periods of time. In studies using inhibitors, LY294002 (50 $\mu \mathrm{mol} / \mathrm{l})$ (Cell Signaling Technology, Danvers, MA, USA), PD98059 (Cell Signaling Technology) (10 $\mu \mathrm{mol} / \mathrm{l})$ or SH-5 (10 nmol/l) were added concomitantly with SDF-1. In studies in which wild-type, dominant-negative or constitutively active forms of kinase were used, dominant-negative TCF7L2, dominant-negative GSK3 $\beta$, constitutively active GSK $3 \beta$, dominant-negative AKT or constitutively active AKT $(0.5 \mu \mathrm{g} /$ well $)$ was co-transfected with TOPflash. Luciferase activity in transfected cells was determined with a luciferase assay kit (Promega, Milwaukee, WI, USA).

SiRNA-mediated knock-down of beta-catenin expression Small interfering RNA (siRNA) fragments against beta-catenin (GenBank accession number NM_053357) were from Dharmacon (Lafayette, CO, USA) (siRNA1 J-100628-05, siRNA2 J-100628-06). siRNAs (50 nmol/l) were transfected into INS-1 cells using Dharmafect reagent. Transfected cells were grown for 48 or $72 \mathrm{~h}$ at $37^{\circ} \mathrm{C}$ in $5 \%$ $\mathrm{CO}_{2}$, then collected or stained for western blot analysis, caspase-3 assay and TUNEL assay, and TOPflash/FOPflash WNT signalling reporter assays.

Western immunoblots Membrane immunoblots were prepared from extracts of INS-1 cells and were interrogated with antisera to beta-catenin (total protein from Santa Cruz Biotechnology, Santa Cruz, CA, USA; active form with the mutated GSK-3 phosphorylation sites from UpState 8E7, UpState, Waltham, MA, USA). Protein density was quantified by densitometric analysis using an image station (440 CF; Eastman Kodak, Rochester, NY, USA).

Thapsigargin or cytokine-induced apoptosis in INS-1 or MIN6 cells For western immunoblot assay (Cell Signaling Technology, Beverly, MA, USA), MIN6 cells were seeded into six-well plates then treated with $1 \mu \mathrm{mol} / 1$ thapsigargin or DMSO control for $16 \mathrm{~h}$, after which apoptotic activity was measured by western immunoblotting using antisera specific for cleaved (active) caspase-3 (9661; Cell Signaling Technology) and cleaved (active) poly ADP ribose polymerase (PARP) (9548; Cell Signaling Technology). For caspase-3 assay, INS-1 cells or MIN-6 cells in 24-well plates were treated with $1 \mu \mathrm{mol} / 1$ thapsigargin or DMSO for $16 \mathrm{~h}$ and caspase-3 activity was determined (Molecular Probes, Eugene, OR, USA). Caspase-3 activity per well was assessed by a microplate fluorescence reader and normalised for total protein with the BCA protein assay (Pierce, Rockford, IL, USA). In studies examining the antiapoptotic effects of SDF-1, $10 \mathrm{nmol} / 1 \mathrm{SDF}-1$ was added concomitantly in the presence or absence of thapsigargin. For cytokine treatment, thapsigargin or DMSO was replaced by cytokine cocktail (10 ng/ml IL- $1 \beta, 50 \mathrm{ng} / \mathrm{ml} \mathrm{TNF}-\alpha$, $50 \mathrm{ng} / \mathrm{ml}$ IFN- $\gamma$ ) or PBS control. In studies where siRNAs were used, beta-catenin siRNA or scramble siRNA were transfected into cells with Dharmafect reagent 1 day (Dharmacon, Lafayette, CO, USA) before thapsigargin treatment.

DAPI (nuclei fluorescence) and TUNEL staining For the TUNEL assay, cells seeded in four-well chamber slides (Lab-Tak) were treated with thapsigargin and TUNEL assay performed using a kit (DeadEnd Fluorimetric TUNEL System; Promega).

Other methods For details on remaining methods, see Electronic supplementary material (ESM).

\section{Results}

SDF-1/CXCR4 axis activates WNT signalling in isolated mouse islets Earlier we reported on production of SDF-1 in MIN6 beta cells and in the peri-islet stromal tissue and intra-islet endothelial tissue of adult mouse pancreas, as well as on the abundance of the SDF-1 receptor, CXCR4, on mouse islet beta cells and the MIN6 and INS-1 clonal beta cell lines [2]. Since SDF-1 was recently reported to 
activate the WNT signalling pathway in rat neural progenitor cells [24], we determined whether SDF-1 activates WNT-signalling in pancreatic beta cells. We used isolated islets prepared from the pancreas of the TOPGAL WNT signalling reporter mouse, in which beta-galactosidase activity (blue colour) indicates activation of the downstream WNT signalling pathway. Addition of SDF-1 $(1 \mathrm{nmol} / \mathrm{l})$ to isolated TOPGAL islets turned them blue (Fig. 1a-d). Since the majority of cells $(>80 \%)$ in islets are beta cells, we conclude that SDF-1 activates WNT signalling in beta cells. The specificity of the interaction of SDF-1 with its receptor CXCR4 is shown by inhibition of the WNT signalling response with the specific CXCR4 antagonist, AMD3100 (Sigma-Aldrich, St Louis, MO, USA), and with pertussis toxin, an inhibitor of Galphai/o (Fig. 1a-d). To confirm that SDF-1 activates transcription of the beta-galactosidase gene, PCR of extracts of the islets was performed to demonstrate corresponding changes in levels of beta-galactosidase mRNA (Fig. 1e).

SDF-1 activates WNT signalling in INS-1 cells via the SDF1 receptor To investigate the mechanisms by which SDF-1/ CXCR4 activates WNT signalling in beta cells, we examined SDF-1 induction of WNT signalling in INS-1 cells using a WNT signalling reporter assay (TOPflash/ FOPflash). The TOPflash and FOPflash constructs contain the luciferase reporter either under the control of consensus TCF7L2-binding sites or mutated TCF7L2-binding sites, respectively. Luciferase activity was measured for $4 \mathrm{~h}$ after addition of $1 \mathrm{nmol} / 1$ SDF-1 (Fig. 2a). SDF-1 activated WNT signalling dose-dependently with maximum responses achieved at $0.4 \mathrm{nmol} / \mathrm{l}$ (Fig. 2b). SDF-1 activation of luciferase activity was antagonised by co-incubation with increasing amounts of the CXCR4 antagonist AMD3100 (Fig. 2c), indicating that the activation of WNT signalling by SDF-1 occurs via the SDF-1 receptor. Compared with WNT3a, SDF-1 activated the TOPFlash reporter in INS-1 cells with similar potency, indicating that SDF-1 and WNT3a both impinge equivalently on betacatenin/TCF7L2 activation of transcription (ESM Fig. 1).

SDF-1/CXCR4 are coupled to the Galphai/o-PI3K-AKTGSK3 $\beta$ signalling pathway The SDF-1 receptor CXCR4 is a G-protein-coupled receptor (GPCR) coupled to pertussis toxin-sensitive Galphai2. Both PI3K/AKT and mitogenactivated protein kinase (MAPK) or ERK kinase (MEK)// ERK1/2 pathways are activated by SDF-1/CXCR4 in HeLa epithelioid carcinoma cells [27]. SDF-1 promotes pancre-
Fig. 1 SDF-1 (1 nmol/l) activates WNT-signalling in isolated islets from the TOPGAL WNT signalling reporter mouse. In comparison to control (a), isolated islets expressed reporter-driven

beta-galactosidase (blue colour) (b), an indicator of activation of the WNT signalling reporter LacZ transgene. Specificity of SDF-1 for its receptor CXCR4 is shown by the inhibition of beta-galactosidase expression by AMD3100 (c), a specific antagonist of CXCR4. d Coupling of the SDF-1/ CXCR4 axis $\mathrm{i}$ to the G-protein, Galphai/o, is shown by inhibition of beta-galactosidase expression with pertussis toxin (PTX). e Polymerase chain reaction showing changes in beta-galactosidase mRNA levels in islets shown above. ${ }^{*} p<0.05$ compared with control a

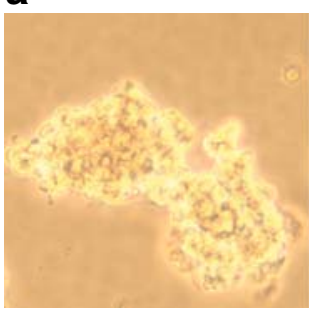

c

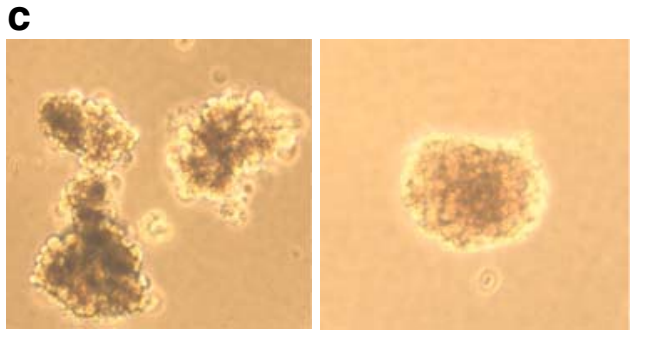

e

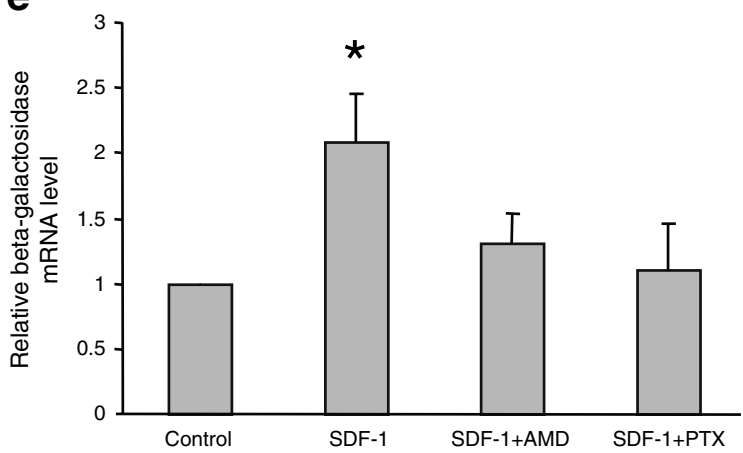

b

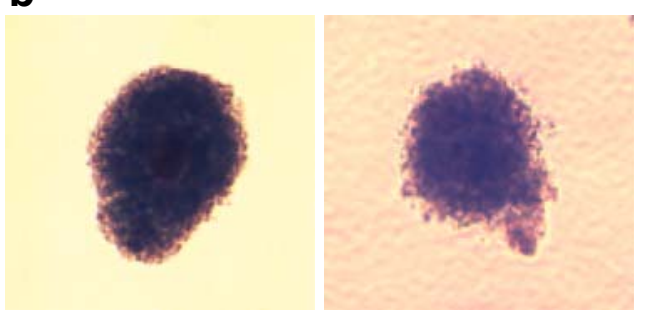

d

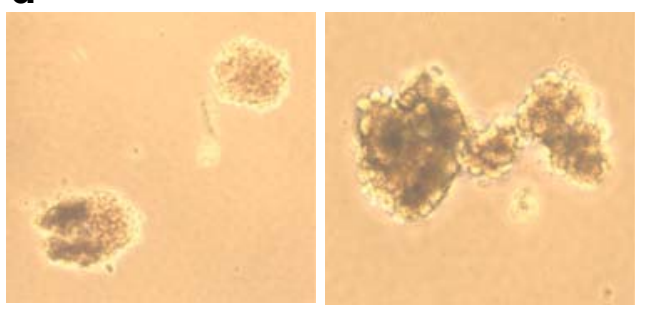

d
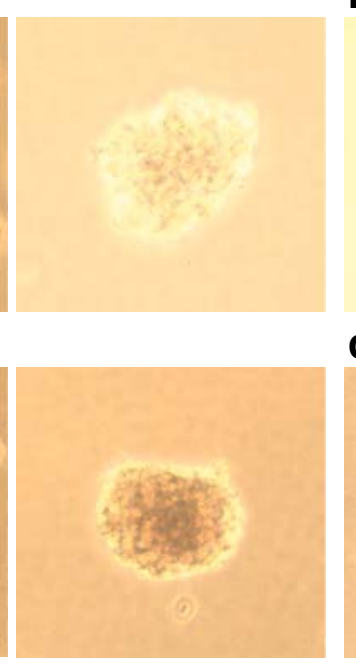

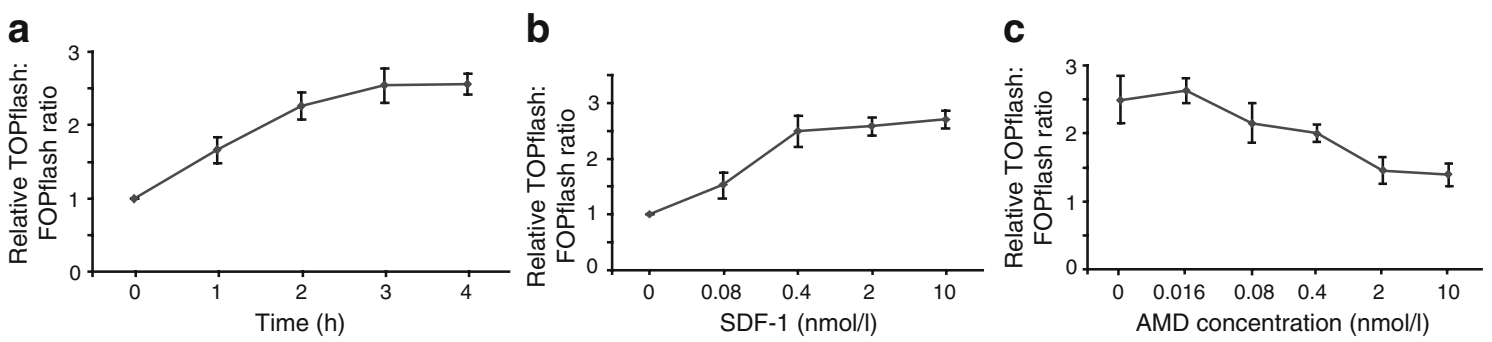

Fig. 2 SDF-1 activates WNT signalling, as indicated by TOPflash luciferase activity:FOPflash activity, in INS-1 cells. a Time course of the TOPflash:FOPflash ratio of INS-1 cells treated with $1 \mathrm{nmol} / \mathrm{l} \mathrm{SDF}$ 1. b Ratio of TOPflash:FOPflash activity in INS-1 cells treated with increasing doses of SDF-1. c Increasing doses of AMD3100 (AMD)

atic beta cell survival in RIP-SDF-1 transgenic mice and in MIN6 and INS-1 clonal beta cells through activation of AKT [2]. Because GSK-3 $\beta$ is inhibited by AKT phosphorylation, which is part of the WNT signalling pathway, we explored whether the activation of PI3K, AKT and inactivation of GSK3 $\beta$ are required for SDF-1-enhanced TOPflash activity. TOPflash reporter assays were performed in INS-1 cells co-transfected with active or inactive forms of GSK3 $\beta$ or pretreated with different kinase inhibitors. Because mutant FOPflash activity was not altered by SDF-1 in repeated experiments, we only measured TOPflash activity in the ensuing experiments. Constitutively active GSK3 $\beta$ inhibited SDF-1-induced TOPflash activity (Fig. 3a), suggesting inactivation of GSK3 $\beta$ is required for SDF-1-induced WNT signalling. Active Galphai/o-PI3K-AKT axis is also required for the effects of SDF-1 on WNT signalling. SDF-1-stimulated TOPflash activity was inhibited by the Galphai inhibitor pertussis toxin, the PI3K inhibitor LY294002 and the AKT inhibitor SH-5 (Fig. 3b) or dominant negative AKT (Fig. 3c). A constitutively active AKT did not augment SDF-1-induced activity, indicating that SDF-1 achieved antagonised $1 \mathrm{nmol} / 1 \mathrm{SDF}-1$ activation of WNT signalling. The $x$-axes for $\mathbf{b}$ and $\mathbf{c}$ are logarithmic scales. All values are relative to the value of untreated INS-1 cells. Data were normalised for transfection efficiency by co-transfected beta-galactosidase and are presented as means \pm SD of three experiments

maximum AKT-mediated effects. In contrast, the activation of (MAPK) was not involved in SDF-1-induced WNT signalling, since the inhibitor of that kinase, PD98059, did not affect SDF-1-stimulated TOPflash activity (Fig. 3b).

TCF7L2 and beta-catenin necessary for SDF-1-mediated WNT signalling The transcriptional activation of WNT target genes requires the association of TCF7L2 with nuclear beta-catenin. Therefore, we investigated whether active TCF7L2 is necessary for SDF-1-induced WNT signalling. INS-1 cells were transfected with a dominantnegative TCF7L2 construct that lacks the beta-catenin interactive domain and thereby inhibits canonical WNT signalling. The expression of dominant-negative TCF7L2 reduced basal and SDF-1 induced TOPflash activity compared with cells transfected with control vector (Fig. 4a). In addition to examining the effects of the inhibition of TCF7L2 on SDF-1-mediated WNT signalling, we examined the requirement for active beta-catenin in this signalling. siRNAs to beta-catenin markedly inhibited basal and SDF-1-mediated TOPFlash activity (Fig. 4b). To investigate whether SDF-1 increases the stability of beta-
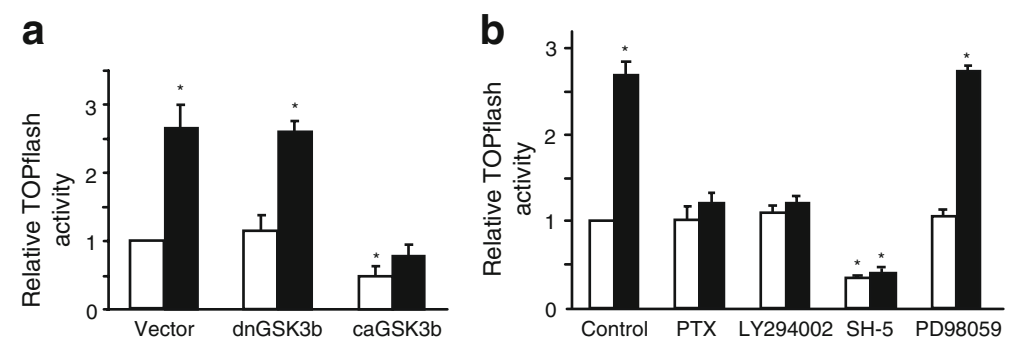

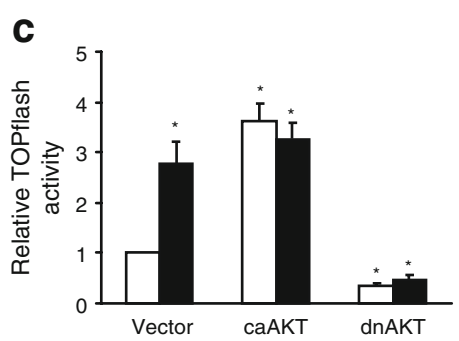

Fig. 3 Roles of PI3K, AKT, epidermal growth factor (EGF receptor) and GSK $3 \beta$ in basal levels of and SDF-1-induced WNT signalling in INS-1 cells. a Constitutively active GSK3 $\beta$ (caGSK3b) inhibited TOPflash activity whereas dominant-negative GSK3 $\beta$ (dnGSK3b) had no effect. b The inhibitors pertussis toxin (PTX) (Galphai), LY294002 (PI3K) and PD98059 (ERK) did not affect basal level TOPflash activity. In contrast, pertussis toxin and LY294002, but not PD98059, did inhibit SDF-1 induced TOPflash activity. The AKT inhibitor SH-5 inhibited basal and SDF-1-induced TOPflash activity. c Constitutively active AKT (caAKT) stimulated basal endogenous WNT signalling (TOPflash activity), whereas dominant-negative AKT (dnAKT) inhibited basal and SDF-1-induced TOPflash activity. All values are relative to the first (leftmost) bar. Data were normalised for transfection efficiency by co-transfected beta-galactosidase and are presented as means $\pm \mathrm{SD}$ of three experiments. White bars, vehicle treatment (control); black bars, $1 \mathrm{nmol} / 1 \mathrm{SDF}-1$ treatment. ${ }^{*} p<0.05$ compared with vehicle treated vector only 


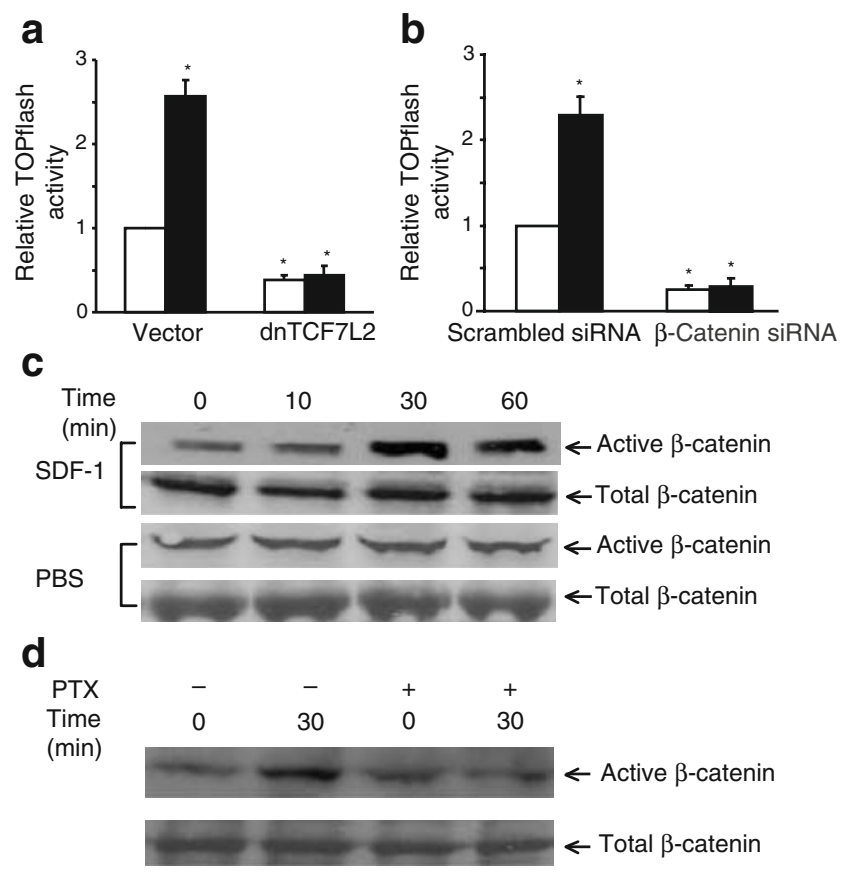

Fig. 4 Inhibition of TCF7L2 or beta-catenin suppresses SDF-1induced TOPflash activity. a Dominant-negative (dn)TCF7L2 inhibits basal (white bars) and SDF-1-induced (black bars) TOPflash activity. b Scrambled siRNAs to beta-catenin siRNAs inhibit SDF-1-stimulated WNT signalling in INS-1 cells. INS-1 cells were transfected and preincubated with TOPflash and the combination of either siRNAs 1 and 2, or scrambled siRNA, and then treated for $4 \mathrm{~h}$ with $1 \mathrm{nmol} / 1 \mathrm{SDF}$ 1 or control vehicle. Data were normalised for transfection efficiency by co-transfected beta-galactosidase and are presented as means \pm SD of three experiments. All values are relative to the first (leftmost) bar. $* p<0.05$ compared with control values (first bar). c Immunoblot analysis with anti-beta-catenin or anti-unphosphorylated beta-catenin (active beta-catenin) showing that SDF-1 stabilised beta-catenin through GPCR-coupled Galphai/o. INS-1 cell cultures were stimulated with SDF-1 for the indicated times. A time-course study showed that SDF-1 $(1 \mathrm{nmol} / \mathrm{l})$ significantly increased the accumulation of cellular betacatenin within $30 \mathrm{~min}$ of stimulation. d Co-incubation of Galphai inhibitor pertussis toxin (PTX) inhibited the accumulation of betacatenin

catenin, the level of active beta-catenin (unphosphorylated on Ser-33 and Ser-37) was examined. An immunoblot of cells treated with SDF-1 for different periods of time was probed with an antibody specific for unphosphorylated beta-catenin. In response to SDF-1, the level of active betacatenin increased 30 min after treatment of INS- 1 cells with SDF-1, whereas PBS treatment had no effect (Fig. 4c, d). Although no changes were observed in cellular levels of total beta-catenin for up to $60 \mathrm{~min}$ of SDF-1 treatment of INS-1 cells, a substantial increase in total beta-catenin was observed after 24 to $48 \mathrm{~h}$ of SDF-1 treatment (ESM Fig. 2b). Pertussis toxin abrogated SDF-1-induced accumulation of active beta-catenin, indicating that stabilisation of beta-catenin by SDF-1 is mediated by a GPCR coupled to Galphai/o (Fig. 4c, d). The semi-quantitative changes for data obtained on western blots is shown in ESM Fig. 3.
SDF-1 attenuation of thapsigargin-induced apoptosis in INS-1 and MIN6 beta cells is mediated by beta-catenin To directly explore the role of downstream WNT signalling in the cytoprotective actions of SDF-1 on beta cells, the clonal INS-1 and MIN6 beta cells were treated with thapsigargin, a potent inducer of endoplasmic reticulum stress-induced apoptosis in beta cells [2]. Apoptosis was measured by western immunoblots that specifically detect the cleaved fragments of caspase-3 and PARP (Fig. 5a). Thapsigargin increased the levels of cleaved caspase- 3 and PARP, which were inhibited by SDF-1. Addition of siRNA to betacatenin, but not scrambled siRNA control, attenuated inhibition of cleaved caspase-3 and PARP (Fig. 5a). The activation of caspase- 3 was also measured to evaluate apoptosis during thapsigargin treatment. Thapsigargin induced caspase-3 activity by threefold, whereas SDF-1 attenuated the pro-apoptotic effect (Fig. 5b, c). We also measured caspase- 3 activity when endogenous beta-catenin expression was inhibited by siRNA. Reduction of betacatenin expression with beta-catenin siRNA partially reversed the anti-apoptotic effects of SDF-1 (Fig. 5b, c). To substantiate these findings, we performed additional apoptosis assay/TUNEL assays (Fig. 5d). Under standard conditions, only a few cells were TUNEL-positive. The numbers of TUNEL-positive cells increased after treatment with thapsigargin. The effect of thapsigargin was inhibited by co-incubation of the cells with SDF-1. The introduction of beta-catenin siRNA, but not scramble siRNA, reversed the cytoprotective actions of SDF-1. The introduction of siRNA alone in INS-1 cells did not induce apoptosis (Fig. 5d and data not shown). These findings indicate that SDF-1 exerts beta-catenin-dependent anti-apoptotic effects on INS- 1 cells.

SDF-1 protects against cytokine-induced apoptosis In addition to thapsigargin, we examined cytokines as inducers of apoptosis, as this is a model more relevant to autoimmunity type 1 diabetes. SDF-1 also inhibited apoptosis (caspase-3 induction) mediated by cytokines $(10 \mathrm{ng} / \mathrm{ml} \mathrm{IL-1} \beta, 50 \mathrm{ng} / \mathrm{ml}$ TNF- $\alpha, 50 \mathrm{ng} / \mathrm{ml} \mathrm{IFN}-\gamma)$ in INS-1 cells (ESM Fig. 4).

SDF-1 does not stimulate proliferation of INS-1 cells In contrast to the GLP-1 agonist, exendin-4, as reported earlier [22], SDF-1 exerts no detectable effects on the proliferation of INS-1 cells as determined by BrdU incorporation and 3(4,5-dimethylthiazol-2-yl)-2,5-diphenyltetrazolium bromide (MTT) assays (Fig. 5e-g).

SDF-1 regulation of gene expression on focused microarrays The expression of transcripts in INS-1 cells in response to SDF-1 $(10 \mathrm{nmol} / \mathrm{l}, 4 \mathrm{~h})$ was determined on focused arrays of genes expressed in the WNT signalling 
a

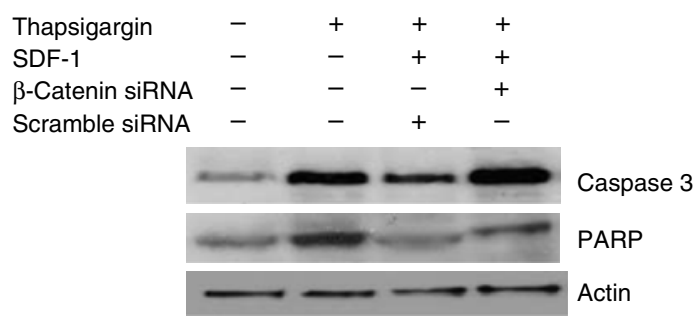

d

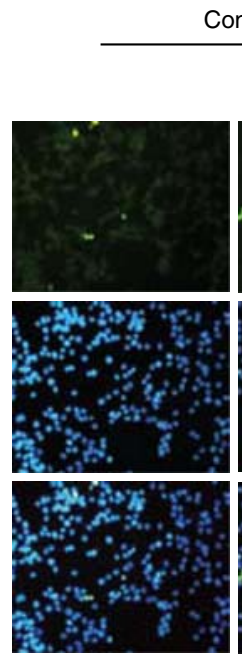

e

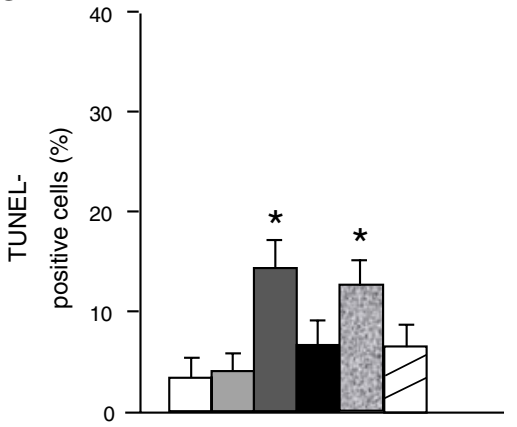

b

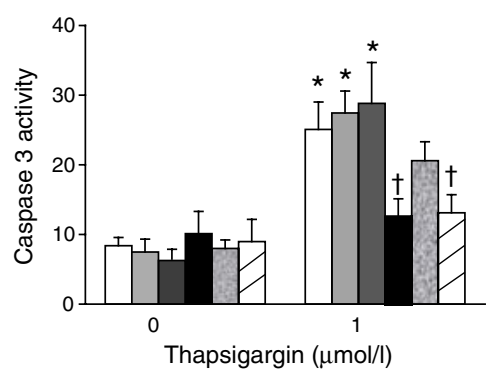

C

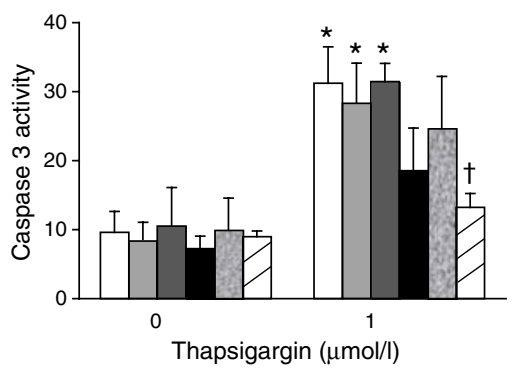

Control

$+\beta$-Catenin siRNA
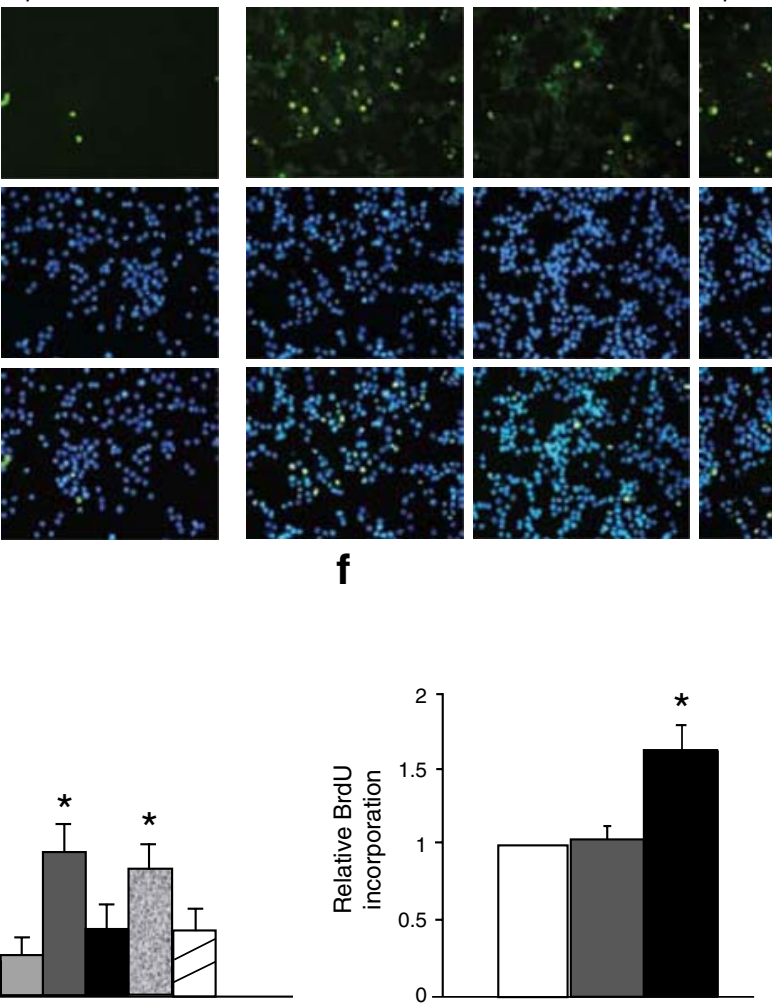

f

Thapsigargin

+ SDF-1
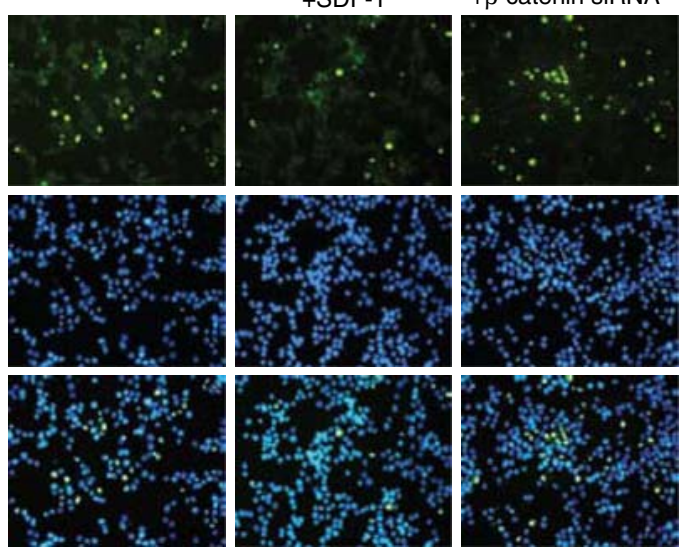

g

+ SDF-1

$+\beta$-catenin siRNA + scramble siRNA
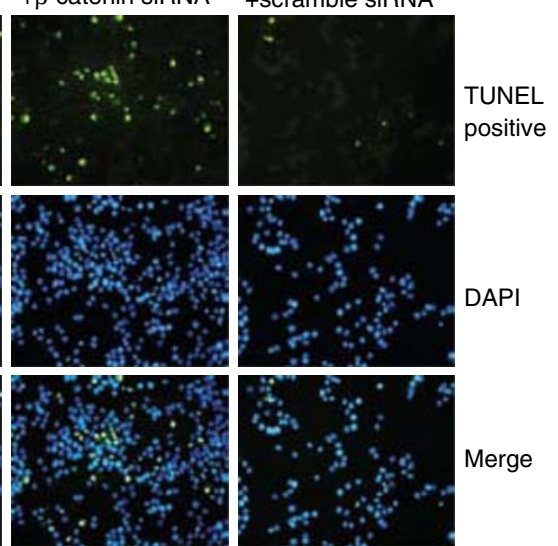

Fig. 5 Inhibition of apoptosis by SDF-1 in INS-1 and MIN-6 cells is reversed by knock-down of beta-catenin. a Western blot analysis of extracts from MIN6 cells that were mock-treated, thapsigargin-treated $(1 \mu \mathrm{mol} / \mathrm{l})$ and/or siRNA-transfected $(0.25 \mathrm{mg} / \mathrm{ml})$ by using anti-sera against cleaved caspase- 3 and cleaved PARP, and actin antibody as a loading control. b Caspase- 3 activity (fluorescence $[\mathrm{mg} \text { protein }]^{-1} \times$ $10^{-5}$ ) of INS-1 or (c) MIN6 cells treated as above (a). Cell treatments from left to right: white bars, control; light grey, beta-catenin siRNA; dark grey, scramble siRNA; black, SDF-1; mottled, SDF-1 and betacatenin siRNA; striped, SDF-1 and scramble siRNA. Results are presented as mean $\pm \mathrm{SD}$ for $n=3$ independent experiments. All values

pathway. Notably, the expression of Ctnnb1 mRNA and protein was substantially upregulated (ESM Table 1, ESM Fig. 5). This finding is consistent with the downregulation of genes expressing components of the beta-catenin destruction box, which is involved in the degradation of are relative to the first (leftmost) bar. ${ }^{*} p<0.05$ compared with untreated control values (leftmost bar); $\dagger p<0.05$ compared with $1 \mathrm{mmol} / \mathrm{l}$ thapsigargin treated control values. $\mathbf{d}$ Representative images of TUNEL staining of INS- 1 cells of $n=3$ independent experiments. e Quantification of apoptotic cells expressed as per cent apoptotic cells relative to total cells counted. $\mathbf{f}$ The proliferation rates of INS-1 cells after SDF-1 (grey bars) or exendin-4 (black bars), presented as relative BrdU and (g) MTT incorporation values compared with controltreated cells. ${ }^{*} p<0.05$ when compared with control values (leftmost bar)

beta-catenin that occurs in the absence of active WNT signalling. Examples of these genes are Axin (also known as Axin1), Gsk3 (also known as Gsk3b) and Csnk-1. These findings are consistent with the mechanism of WNT/ frizzled-induced signalling involving Galphai/o activation 
of $D v l$ (also known as Dvll) and inhibition of the destruction box enzymes GSK3 and Csnk1 that destabilise and degrade beta-catenin. Notably, SDF-1 resulted in the downregulation of $C c n d 2$ and $M y c$, both involved in cell cycle progression (ESM Fig. 5, ESM Table 1). Ccndl was upregulated by SDF-1 (ESM Fig. 5, ESM Table 1), confirmed by real-time quantitative PCR (ESM Fig. 2a), suggesting that the failure of SDF-1 to stimulate INS-1 cell proliferation (Fig. 5e-g) is due to a lack of cyclin D2, and not of cyclin D1.

Regulation of the expression of different cyclins in beta cells is complex. Of note, it has been shown that GLP-1 agonists enhanced expression of cyclinA2 in beta cells [28].

\section{Discussion}

In the present studies we tested the hypothesis that SDF-1 activates WNT signalling in pancreas-derived INS-1 beta cells and isolated islets ex vivo. These studies provide novel evidence that SDF-1 enhances WNT signalling in beta cells and isolated islets. Further, we found that SDF-1 activates WNT signalling by binding to its receptor CXCR4, a G-protein-coupled receptor that activates Galphai and the downstream PI3K-AKT axis, resulting in the subsequent deactivation of GSK3 $\beta$ and stabilisation of beta-catenin (Fig. 6). The mechanisms of SDF-1-mediated WNT signalling differ from those of GLP-1 agonists reported earlier [22] in that GLP-1-activated protein kinase A (PKA) directly stabilises beta-catenin independently of PI3K/AKT and GSK3 (Fig. 6).

A functional role of beta catenin and TCF7L2 in SDF-1mediated survival and cytoprotection of beta cells was demonstrated by knock-down of beta-catenin with siRNAs. siRNAs antagonised the SDF-1-mediated inhibition of thapsigargin-induced beta cell apoptosis, indicating that WNT signalling is obligatory to the prosurvival effects of SDF-1. Modulation of WNT signalling regulates apoptosis in cancer cell lines and primary cells [20]. WNT3a and WNT5a promote proliferation and inhibit apoptosis in HEK293 cells [29]. Anti-WNT-1 siRNA inhibits WNT signalling and induces apoptosis in human breast cancer MCF-7 cells [30]. Increased expression of beta-catenin increases proliferation and inhibits apoptosis of vascular smooth muscle cells following carotid injury in SpragueDawley rats [31].

Luo et al. reported that in rat hypothalamus SDF-1 stabilised cytoplasm levels of beta-catenin and induced both beta-catenin translocation into the nucleus and binding to TCF7L2, as well as activating transcription of proproliferative genes such as Ccnd1, Ccnd2, Ccnd 3 and the transcription factor $M y c$ [24]. Our findings reported here
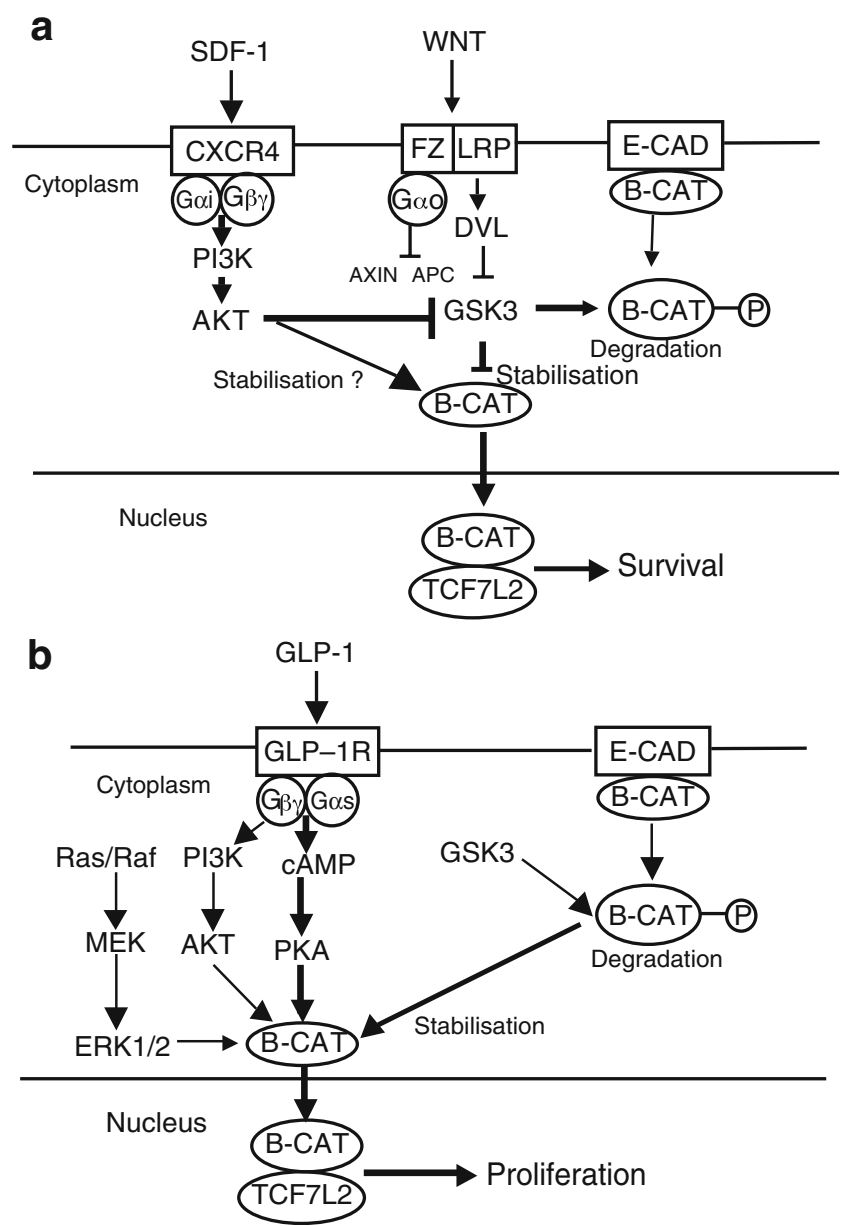

Fig. 6 Schematic models of signalling pathways used by SDF-1/ CXCR4 and GLP-1/GLP-1R in the activation of beta catenin/ TCF7L2-mediated transcriptional expression of genes involved in beta cell survival and proliferation a SDF-1 activation of WNT signalling. Upstream SDF-1/CXCR4 actions are transmitted to betacatenin/TCF7L2 predominantly via action of PI3K/AKT, the inhibition of GSK3 by AKT and the resultant stabilisation of beta catenin. Beta-catenin/TCF7L2 activated by SDF-1 results in the transcription of genes involved in cell survival. A direct action of AKT on the stabilisation of beta-catenin has been suggested [34, 35], but remains conjectural. b GLP-1 activation of WNT signalling. Upstream GLP-1/ GLP-1R actions are transmitted to beta-catenin/TCF7L2 via cAMP/ PKA and the direct stabilisation of beta-catenin by PKA. PI3K/AKT and GSK3 are minimally or not at all involved in the GLP-1-mediated pathway. The actions of beta-catenin/TCF7L2 mediated by GLP-1 are primarily to activate the transcription of genes responsible for cell proliferation. APC, adenomatous polyposis coli; AXIN, axis inhibition protein; B-CAT, beta-catenin; E-CAD, epithelial cahedrin; DVL, dishevelled, dsh homologue $1 ; \mathrm{G}_{\beta \gamma}, \mathrm{G}$ protein beta subunits; $\mathrm{G}_{\alpha \mathrm{o}}, \mathrm{G}$ protein alpha unit o; G $\alpha \mathrm{s}$, G protein alpha unit s; G $\alpha$ i, G protein alpha unit i; FZ, frizzled; LRP, low-density lipoprotein receptor-related protein; MEK, mitogen activated protein kinase; $\mathrm{P}$, phosphorylation; Raf, v-raf-1 murine leukaemia viral oncogene homologue 1; Ras, rat sarcoma viral oncogene homologue

show that the coupling of SDF-1/CXCR4 signalling to downstream WNT signalling involves Galphai/o as a major coupling component and that Galphai/o is an activator of PI3K, which in turn activates the prosurvival kinase AKT. 
We also found that SDF-1 induces transcription of betacatenin in INS-1 cells. In contrast to the well-defined mechanism that accounts for the post-translational regulation of beta-catenin, the transcriptional regulation of betacatenin is less well studied. SDF-1 is among the few ligands that have been shown to activate beta-catenin gene transcription. Our discovery raises the interesting possibility of a biphasic upregulation of WNT and beta-catenin signalling by SDF-1, achievable acutely by post-translational modifications and in the longer term by de novo synthesis of beta-catenin.

The involvement of WNT signalling and beta-catenin in pancreas development remains unclear. The expression of a frizzled receptor antagonist in pancreatic progenitor cells of transgenic mice resulted in a $75 \%$ reduction in overall pancreatic mass and a 50\% reduction in absolute beta cell numbers [32]. Most recently, human islet-derived precursor cells (hIPCs) have been shown to exhibit intense betacatenin nuclear staining, an indicator of activated WNT signalling [33]. hIPCs exhibit nuclear beta-catenin throughout exponential cell growth. Beta-catenin signalling is indispensable for hIPC proliferation and during hIPC derivation from islets. Coincidently, we discovered that inhibition of TCF7L2, an obligatory binding partner of beta-catenin, suppressed proliferation of INS-1 cells and resulted in a reduction in beta cell mass [22]. TCF7L2 appears to be required for maintaining glucose-stimulated insulin secretion and beta cell survival, since genetic studies in humans have identified a close association of polymorphisms in TCF7L2 and susceptibility towards type 2 diabetes [23]. Our findings indicate that WNT activation is required for the proproliferative function of GLP-1 agonists and the prosurvival functions of SDF-1 in pancreatic beta cells.

Dissimilarities and similarities between SDF-1 and GLP-1mediated WNT signalling in beta cell These studies of SDF-1 actions and earlier studies of GLP-1 actions [22] on beta cells demonstrate that both SDF-1 and GLP-1 activate downstream WNT signalling via beta-catenin/TCF7L2regulated gene transcription. Here we show that downstream WNT signalling is required for the anti-apoptotic actions of SDF-1, having reported earlier the requirement of WNT signalling for the proproliferative actions of GLP-1 [22]. Notably, in the studies reported here, we found no detectable effects of SDF-1 on beta cell proliferation (Fig. 5e-g).

There are differences between the interactions of SDF-1/ CXCR4 signalling and GLP-1/GLP-1 receptor (GLP-1R) signalling with the WNT signalling pathway in beta cells. Although SDF-1 and GLP-1 activate the downstream pathway of WNT signalling, consisting of beta-catenin/ TCF7L2-mediated gene expression, they do so by different pathways of interactions with the more upstream components of the WNT signalling pathway. SDF-1 inhibits the destruction box of the canonical WNT signalling pathway consisting of axis inhibition protein (AXIN), adenoma polyposis coli, and the protein kinases GSK3 and casein kinase-1 (CSNK1). This inhibition of GSK3 and Csnk1 by SDF-1 is likely to be mediated by the well-known actions of AKT in inhibiting these kinases, resulting in the stabilisation and accumulation of beta-catenin [11]. In marked contrast to the actions of SDF-1 on beta cells, GLP-1 activates beta-catenin/TCF7L2 complexes via the stabilisation of beta-catenin by a different mechanism involving phosphorylation and stabilisation of beta-catenin by the cAMP-dependent PKA. PKA activated by GLP-1/ GLP-1R phosphorylates beta-catenin on Serine-675, resulting in its stabilisation and accumulation. Thus, unlike SDF-1, GLP-1-induced activation of gene expression by beta-catenin/ TCF7L2 in beta cells occurs independently of the destruction box and the activities of GSK3 [22]. It also remains possible that beta-catenin may be stabilised by its direct phosphorylation by $\operatorname{AKT}[33,34]$

Notably, beta-catenin and TCF7L2 comprise the components of a non-covalent bipartite transcriptional activation complex. Beta-catenin is the activation domain and TCF7L2 is the DNA-binding domain of the transactivator. We speculate that different phosphorylation forms of betacatenin provided by SDF-1 signalling vs GLP-1 signalling result in different conformations of beta-catenin. When different conformers of beta-catenin interact with TCF7L2, they confer different conformations to the DNA-binding domains of TCF7L2, resulting in differing affinities of TCF7L2 for its cognate enhancer binding sites on the promoters of various WNT signalling target genes. Such a combinatorial mechanism could account for the difference in genes regulated by beta-catenin/TCF7L2 in beta cells in response to SDF-1 compared with GLP-1 (ESM Fig. 5) [22].

WNT-signalling may be a final downstream pathway for SDF-1 and GLP-1 signalling in beta cells. However, gene expression targets diverge so that SDF-1 predominately regulates genes involved in cell survival, whereas GLP-1 regulates genes involved in cell cycle control (proliferation). If this circumstance proves to be valid, our findings raise the possibility of a dual therapeutic approach for increasing beta cell mass. GLP-1 is predominantly progrowth and SDF-1 is predominantly pro-survival. Thus the two peptides may act additively or synergistically to promote both growth and survival of beta cells, and to conserve or even enhance beta cell mass in response to injury.

Acknowledgements We thank T. Boodry and S. Avadhani for technical assistance, and V. Stanojevic and M. Thomas for helpful suggestions during the studies. This work was supported in part by a postdoctoral fellowship from the Juvenile Diabetes Research foundation (to Z. Lui) and a grant USPHS DK30834 (to J. F. Habener). 
Duality of interest The authors declare that there is no duality of interest associated with this manuscript.

\section{References}

1. Butler AE, Janson J, Bonner-Weir S, Ritzel R, Rizza RA, Butler PC (2003) Beta-cell deficit and increased beta-cell apoptosis in humans with type 2 diabetes. Diabetes 52:102-110

2. Yano T, Liu Z, Donovan J, Thomas MK, Habener JF (2007) Stromal cell derived factor-1 (SDF-1)/CXCL12 attenuates diabetes in mice and promotes pancreatic beta-cell survival by activation of the prosurvival kinase AKT. Diabetes 56:2946-2957

3. Burger JA, Kipps TJ (2006) CXCR4 a key receptor in the crosstalk between tumor cells and their microenvironment. Blood 107:1761-1767

4. Kucia M, Ratajczak J, Ratajczak MZ (2005) Bone marrow as a source of circulating CXCR4+ tissue-committed stem cells. Biol Cell 97:133-146

5. Ratajczak MZ, Zuba-Surma E, Kucia M, Reca R, Wojakowski W, Ratajczak J (2006) The pleiotropic effects of the SDF-1-CXCR4 axis in organogenesis, regeneration and tumorigenesis. Leukemia 20:1915-1924

6. Kryczek I, Wei S, Keller E, Liu R, Zou W (2007) Stroma-derived factor (SDF-1/CXCL12) and human tumor pathogenesis. Am J Physiol Cell Physiol 292:C987-C995

7. Schober A, Karshovska E, Zernecke A, Weber C (2006) SDF1alpha-mediated tissue repair by stem cells: a promising tool in cardiovascular medicine? Trends Cardiovasc Med 16:103-112

8. Dalakas E, Newsome PN, Harrison DJ, Plevris JN (2005) Hematopoietic stem cell trafficking in liver injury. FASEB J 19:1225-1231

9. Nagasawa T, Hirota S, Tachibana K et al (1996) Defects of B cell lymphopoiesis and bone-marrow myelopoiesis in mice lacking the CXC chemokine PBSF/SDF-1. Nature 382:635-638

10. Zou YR, Kottmann AH, Kuroda M, Taniuchi I, Littman DR (1998) Function of the chemokine receptor CXCR4 in haematopoiesis and in cerebellar development. Nature 393:595-599

11. Welters HJ, Kulkarni RN (2008) WNT signalling: relevance to beta cell biology and diabetes. Trends Endocrinol Metab 19:349355

12. Kayali AG, van Gunst K, Campbell IL et al (2003) The stromal cell-derived factor-1alpha/CXCR4 ligand-receptor axis is critical for progenitor survival and migration in the pancreas. J Cell Biol 163:859-869

13. Ide A, Kawasaki E, Abiru N et al (2003) Stromal-cell derived factor-1 chemokine gene variant is associated with type 1 diabetes age at onset in Japanese population. Hum Immunol 64:973-978

14. Dubois-Laforgue D, Hendel H, Caillat-Zucman S et al (2001) A common stromal cell-derived factor-1 chemokine gene variant is associated with the early onset of type 1 diabetes. Diabetes 50:1211-1213

15. Aboumrad E, Madec AM, Thivolet C (2007) The CXCR4/ CXCL12 (SDF-1) signalling pathway protects non-obese diabetic mouse from autoimmune diabetes. Clin Exp Immunol 148:432439
16. Moon RT, Kohn AD, de Ferrari GV, Kaykas A (2004) WNT and beta-catenin signalling: diseases and therapies. Nat Rev Genet 5:691-701

17. Moon RT (2005) WNT/beta-catenin pathway. Sci STKE 2005: $\mathrm{cm} 1$

18. Tian Q, Jin H, Cui Y, Guo C, Lu X (2005) Regulation of WNT gene expression. Dev Growth Differ 47:273-281

19. Nelson WJ, Nusse R (2004) Convergence of WNT, beta-catenin, and cadherin pathways. Science 303:1483-1487

20. Logan CY, Nusse R (2004) The WNT signaling pathway in development and disease. Annu Rev Cell Dev Biol 20:781-810

21. Gordon MD, Nusse R (2006) WNT signaling: multiple pathways, multiple receptors, and multiple transcription factors. J Biol Chem 281:22429-22433

22. Liu Z, Habener JF (2008) Glucagon-like peptide-1 activation of TCF7L2-dependent WNT signaling enhances pancreatic beta cell proliferation. J Biol Chem 283:8723-8735

23. Florez JC (2008) Newly identified loci highlight beta cell dysfunction as a key cause of type 2 diabetes: where are the insulin resistance genes? Diabetologia 51:1100-1110

24. Luo Y, Cai J, Xue H, Mattson MP, Rao MS (2006) SDF1alpha/ CXCR4 signaling stimulates beta-catenin transcriptional activity in rat neural progenitors. Neurosci Lett 398:291-295

25. Lacy PE (1994) Pancreatic islet cell transplant. Mt Sinai J Med 61:23-31

26. DasGupta R, Fuchs E (1999) Multiple roles for activated LEF/ TCF transcription complexes during hair follicle development and differentiation. Development 126:4557-4568

27. Peng SB, Peek V, Zhai Y et al (2005) AKT activation, but not extracellular signal-regulated kinase activation, is required for SDF-1alpha/CXCR4-mediated migration of epitheloid carcinoma cells. Mol Cancer Res 3:227-236

28. Song WJ, Schreiber WE, Zhong E et al (2008) Exendin-4 stimulation of cyclin A2 in beta-cell proliferation. Diabetes 57:2371-2381

29. Jia L, Miao C, Cao Y, Duan EK (2008) Effects of WNT proteins on cell proliferation and apoptosis in HEK293 cells. Cell Biol Int 32:807-813

30. Wieczorek M, Paczkowska A, Guzenda P, Majorek M, Bednarek AK, Lamparska-Przybysz M (2008) Silencing of WNT-1 by siRNA induces apoptosis of MCF-7 human breast cancer cells. Cancer Biol Ther 7:268-274

31. Wang X, Xiao Y, Mou Y, Zhao Y, Blankesteijn WM, Hall JL (2002) A role for the beta-catenin/T cell factor signaling cascade in vascular remodeling. Circ Res 90:340-347

32. Papadopoulou S, Edlund H (2005) Attenuated WNT signaling perturbs pancreatic growth but not pancreatic function. Diabetes $54: 2844-2851$

33. Ikonomou L, Geras-Raaka E, Raaka BM, Gershengorn MC (2008) Beta-catenin signaling in mesenchymal islet-derived precursor cells. Cell Prolif 41:474-491

34. Tian Q, Feetham MC, Tao WA et al (2004) Proteomic analysis identifies that 14-3-3zeta interacts with beta-catenin and facilitates its activation by AKT. Proc Natl Acad Sci U S A 101:1537015375

35. Fang D, Hawke D, Zheng Y et al (2007) Phosphorylation of betacatenin by AKT promotes beta-catenin transcriptional activity. J Biol Chem 282:11221-11229 